\title{
Index
}

Note that only parts of the human body are systematically indexed here. The terms 'ekphrasis' and 'physiognomy' appear on nearly every page of the volume and only a few occurrences are included here.

A capite ad calcem $82,83,132$

Abscess, boil 76

Abū Bakr al-Rāzī 412, 436

Abū Ma'shar 376

Achaemenids 251

Achilles 176

Achilles tendon 103

Actio 183, 195, 197, 198

Adab hymns 4

Adad-apla-iddina 43, 82

Adamantius 257, 260, 261

Addressee 26

Aelius Aristides 255

Aelius Ligus 234

Aelius Theon 5, 143, 157, 231, 243

Aesop 191

Aethiopians 260

Aetiology 71

Affections (of the soul) 147

Affective/emotive manifestations 242

Afghanistan 42, 58

Against Aristogeiton 143

Against the Sophists 184

Agamemnon 171

Ahû-tablet 45, 47, 48

Airs, Waters, Places 234, 245, 246

Ajax 171

Akkadian 49

al-Alqami 360

Alamdimmû 44, 47, 53, 58, 81, 82, 84, 85, 86 , $106,107,108,109,113,120,130,131,132$

Alans 259, 263, 264

al-Bisțāmī 423

Alchemical 333

Alcidamas 184

al-Dimashqī 7, 447, 448, 452

Alexander (the Great) 251, 331, 337, 375, 401

al-Farabi 364

Allegoriae lliadis 176, 177

Allusive 243

al-Maqāmāt al-falsafiyya wa-l-tarjamāt al-șūfiyya ("Philosophical sessions and sufic interpretations") 448

al-Rāzī 336
al-Riyāsa fĩ̄ilm al-firāsa 447

Alternations between first, second and third person 26

al-Ṭibb al-Manșūrī 393

Ālu ina mēle šakin 43

Ammianus Marcellinus 213, 215, 225, 257, 258, 259, 261, 262, 264

Analogies 245, 267

Anat 301

Anatolia 57

Anatomo-pathognomic method/modality 193, 195, 196

Anger 188

Animal 145, 236, 238, 241, 336

Animal analogies 242

Animal fables 250

Animal for zodiacal sign 134

Animal(s) of 13 and 277135

Animal omens 115

Animal-like passion 281

Animated statue 14

Ankle $66,68,70$

- thick 242, 274, 341, 399, 421

- thin 341, 399

Anomymus Latinus 192, 217, 220, 222

Antehomerica 177

Antenor 171

Antiquarian 254

Antonomasia 162

Anus 74, 76, 104

Anzu(d)-bird 6, 33, 34, 35

Anzuzu-spider 105

Aphorisms of the Philosophers

(Ādāb al-falāsifa) 371

Aphrodisias Sebasteion 251, 256

Aphthonius of Antioch 5,157

Apollonius of Tyana 377

Apotelesmatika 237

Appeal to emotions 233

Appearance of fungus 116

Apsasû-bovine 101

Aquarius 128

Arabic 248, 375

Arabic scientific tradition 1 
Aramaic 137

Archelaos of Alexandria 451, 452

Architecture 296

Areolar tissue of the breast 76

Argument 251, 255

Aries 122, 123, 130, 131

Aristotle $6,143,146,148,149,150,152,157$, $161,183,184,185,187,192,196,232,241$, $248,250,331,333,337,369,401,435$

Ark, the 309

Arms 199, 336, 399

Arrogance 173

Artaxerxes II 155

Artemidorus 164

Artemidorus of Daldis 240

Ashurbanipal's Library 86

Asia Minor 250, 256, 259, 268

Āšipu 43, 57

Aššur 83

Assurbanipal 67

Assurbanipal's library 58

Astonishment (ekplexis) 151

Astral influences 236

Astral Science 42, 47

Astrological 239, 260

Astrological determinism 247

Astrological medicine 119, 123

Astrology 119, 121, 238

Astro-medical zodiac scheme 124

Athenians 259

Athirat 301, 302

Atrids 176

Atticus 210

Audience-oriented description 28

Augustus 215, 217, 218, 220, 221

Aulus Gellius 240

Authoritative 252

Authority 251

Avicenna 369

Āyurveda 42

Baal cycle 300, 301, 302

Babylon 122

Babylonian Iatromathematical Calendar 125 , 135, 136

Babylonians 57

Bacchic mania 280

Back 336, 398

Bacon, Roger 337, 371, 373, 436
Bad looking 171

Balinas 377

Baluchistan 58

Barbarians 232, 259

Bardaisanites 247

Bardesanes (or Bardaișan) of Edessa 247

Beard(s) 102, 165, 168, 263, 397

Bearing 233

Beauty 168, 173

Behavioural omens 115

Belly 51, 66, 89, 126, 127, 291, 326, 336, 337 $388,389,398$

- delicate 339

- gracious $377,398,420$

- hairy 329, 373, 395, 417, 458, 459

- large/big 339, 377, 398, 420

- protruding 214

- small 420

- soft 339

- thick 420

- thin 398

Bhavișya 48

Bhavișya Purāṇa 48, 49

Biography 205, 206, 207, 210, 211, 212, 213 , 214, 217, 221, 257

Bison 101

Black bile 274

Blemishes 48, 49, 50

Bloodletting 245

Bodily lines 51

Bodily movement/deportment 47

Bodily postures 194

Body and soul affect each other 146, 194

Body depiction 201

Body Description Texts 19, 20, 23

Boils 49

Book of Politics in the Organisation of the Government (Kitāb al-Siyāsa fĩ tadbīr al-riyāsa) 370

Book of the Crown (Kitāb al-Iklīl) 370

Book of the Orphan, The 376

Book of the Secret of Creation (Kitāb Sirr al-Khalīqa) 377

Book of the Selected invocations and Experienced remedies (Kitāb al-ad'iya al-muntakhaba wa-l-adwiyya al-mujarraba) 423

Book of the Ten Sciences (Liber Decem Scienciarum) 371 
Book of Treasures 378

Boundaries between verbal and visual expression 292

Brahmajāla Sutta 58

Brahmanic lore 55

Braveness 146, 171

Breast 63,69

- broad 460

- delicate 340

- hair on the 329, 373, 395, 458

- not too wide 339

- of pregnant woman 78

- of woman $63,69,72,76$

- tip of 72, 75, 76

Brethren of Purity 366

Ḅ̣hatsaṃhitā 42, 48, 51, 55

Briseis 176, 176

Brutus 183

Brutus, L. 209

Building inscriptions 25

Bull 48

Caelius Aurelianus 274

Calendrical forerunner to the zodiac scheme 126

Calendrical melothesia 125, 126

Caligula 205, 221, 222, 223

Callistratus 155, 156, 157, 278, 280, 283

Calves 399

Canaanite mythic tradition 297

Canon of Medicine 369

Carakasaṃhitā 42

Castor 176

Cat $48,101,103$

Catilina 206

Causal chains 3

Causal inference 192

Causation 238, 239

Celt 241

Celtic 242, 245, 248, 254

Centipede 110, 104

Character (only indexed if qualified in some way)

- bad 337, 340, 398, 460

- cruel 221

- desirous 459

- excellent 210

- future 55

- good 336, 410, 459

- lascivious 221
- licentious 218

- mad / of madness 272, 273, 275

- present 55, 56

- quarrelsome 340

- vile 218

- virtuous 187

Characteristics of peoples and provinces 241

Characterological traits 283

Character types 193,194

Cheeks 20, 21, 68, 154, 155, 171, 198, 387, 397

- bearded 290

- easy 336

- fleshy 335

- furrowed 261

- long 101

- prominent 404,415

- red 278

- seized 89

- slack 242

- smooth 342, 401, 408, 421

- soft 242, 397

- spasms of 273

- swollen 400, 419

Cheerful 165

Chest $34,126,163,172,229,388,389,398$

- broad 12, 171, 217, 218, 399, 408

- hairy 395,417

- large 273, 377, 420

- narrow 420

- prominence 398

- small 420

- straight 197

- wide 398

Childbirth 187

Chin 68

- hairless 460

- loose 101

- long 128

- narrow 415

- turned 460

Choleric type 274

Cicero 1, 152, 183, 184, 185, 188, 192, 194, 197, $198,199,201,209,210,217,233,234$. 240,262

City 268

Civil 18,37

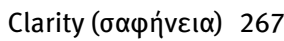

Classical Sumerian literature 11, 12, 14, 30, 36, 37

Classicism 255 
Classifications 251

Claudia 212

Claudius Ptolemy 237

Clause-initial nominal phrases 19

Clear 254

Climate 235, 236

Climatic explanations 238

Climatological models 2, 239, 261

Clothing 233

Cockerel 242

Codicology 358

Codification of manliness 199

Commentary on the Theriac 436

Commentary on Zeno's Epistle 364

Common knowledge 239

Common sense physiognomics 205, 206, 210, 211, 212, 216

Compendium theologicae veritatis 338

Complexion 220, 221, 223

- blue (purple) 415, 416

- dark 233, 257

- fair 409, 415, 421

- light 233

- olive 171

- pale 217, 219, 220, 221, 281, 410, 411, 415

- ruddy/reddish 166, 410, 422

- sallow 415

- white/clear 218, 400, 415, 422

Conditional 46, 54

Coniectatio 240

Constant physical agitation 273

Contempt 188

Convulsive mobility 273

Corax the Sicilian 185

Corinth 259

Cornelius Nepos 210

Corporeal 268

Corpus Aristotelicum 144

Correct behaviour of the king 332

Cosmic corporeality 130

Cryptic letters 132

Cultural hierarchies 240

Cultural polemics 234

Cuneiform writing 16

Curling of the bodily hairs 48

Curling of the hairs (of the head) 52,55

Cutaneous eruptions 49

Dares (Daretes) of Phrygia 161, 162, 164, 174, 176

Dead Sea Scrolls 131, 132
De Anima 147

Decadent 234

Deciphering of the body 196

Decorum 199

De excidio Troiae 176

Deity names 91

De Inventione 183, 184

Demosthenes 143, 210

Depressed 281

De oratore 183

De Physiognomonia Liber 192, 244

Description of women (Wașfal-nisā') 412

Descriptions of Statues 155

Descriptive paradigms $1,11,18$

Determination of suitable partners in marriage 55

Determinism 239

Deuteronomistic history 309

Dharmaśāstra 42

Diagnostic gaze 267

Diagnostic Handbook 85, 92, 110

Diagnostic omens 115

Dictys of Crete 162, 164

Dio Chrysostom 164

Diodorus Siculus 261, 262

Diomeda 176

Direct address to the votive object 5

Diversity 239

Divination 62, 119

Divine manifestation 34

Diviner 50

Diviner's Manual 62

Dodekatemoria 129, 133, 134

Dog 101, 103

Dolon 171

Domestic life 55

Domestic tranquillity 56

Dream 29

Drusus 218

Druze 366

Duck 48

Dumuzi 123

Duties of empire 33

Ea 295

Ears(s) 20, 64, 69, 77, 103, 165, 169, 300, 310, 312, 313, 336, 435

- brown 71

- calloused (like wrestler) 165

- damaged 169 
- flabby 336

- green 71

- large/big 128, 336, 397, 419

- red 71

- ringing 110

- small 336, 376, 397, 419

- yellow 71

- wide 12

Early Dynastic plant compendia 17

Eastern Kufic 359

Eastern Mediterranean 6, 290

Ebla 3

Economic status 55

Ecumenical 250

Egypt(ians) 241, 242, 259, 260, 261

Egyptian priests 240

Eight basic marks 47

Eikos 186

Ekphrasis (description) 1-7, 11-37, 143-158, $162,165,203,215,227-266,275,276$, 280, 285-317, 347-433

Ekphrastic contagion 267

Ekphrastic hope 319

Elephant 48

Elite(s) 230, 243, 266

Elogia 210

Elogium Scipionis 212

Eloquent body 195

Emotion (pathos) 11, 243, 248, 264

Emperor(s) 216, 217, 222

Emperor-biographies 205

Emperor-portraits 215

Empire, the 240

Enargeia (vividness) 11, 13, 148, 150, 151, 152, 231, 233, 236, 247, 266, 267, 277, 283

Encomium of Helen 186

Ennoema 160

Enthymeme 187, 192, 239

Enūma Anu Enlil 43

Enūma Eliš 22, 106, 130

Enumeration 1, 16, 18, 19, 36

Environmental determinism 234, 235, 237

Ephesus 256

Epic of Gilgamesh 292

Epideictic 266

Epistemic appeal 251

Epistemic template 234

Epistles of the Brethren of Purity 437

Epistles of the Sages (Rasā'il al-ḥukamā') 360

Esagil-kin-apli 43, 57, 63, 81
Esagil-kīn-apli text catalogue 82

Esoteric Babylonian Commentary 130

Essential characteristics 252

Essentialising 236, 237, 247, 254, 265, 268

Essentialism 238

Ethnic 245, 254

Ethnic analogies 242

Ethnic customs 248, 256

Ethnic groups 336

Ethnic mockery 255

Ethnic practices 248

Ethnic stereotypes 268

Ethnicised 232, 247, 253, 254

Ethnographic gaze 229

Ethnographical disposition 229

Ethnographical writing 229

Ethnographicising writing 237

Ethnographicising register 229

Ethnography 229, 241

Ethnological method 193

Ethopoeia 232

Ethopoeic 240, 268

Eulogy 210

Euphantasiotos 151, 152

Eusebius' Praeparatio Euangelica 248

Evidentia 152

Exempla 230

Exodus 25-31, 35-40 306

Exorcist 43, 57

Exorcist's Handbook 43, 58

Explanations of micro-movements 200

Expositio totius mundi et gentium 261

Extraneous (ahû) 121

Extra-serial 83

Eyes (only indexed if qualified in some way)

- able to see at night 217

- beautiful 33

- black 155, 165, 171, 233, 259, 330, 374, 395, 408, 409, 416

- blazing 216

- blinking constantly 333

- bloodshot 279

- blue 233, 244, 247, 323, 330, 331, 332, 371, 374, 379, 381, 394, 396, 404, 408, 409, 410, 411, 415, 459

- blue-black 332, 343, 372

- blue-grey 166,170

- brilliant 242

- cow-like 330, 331, 374, 396, 416

- Celtic 244 
- charming 211

- collared 331

- crab-like 332

- dark 247, 330, 374, 396, 416, 421

- delightful 155

- deep(-set) 330, 396, 399, 408, 410

- distressed 279

- drooping 273

- dry 273

- dull 278, 280

- dusky 259

- fast/agile 330, 332

- fierce 168, 247, 260

- fiery 173

- filled with anxiety 279

- fixed 279

- flashing 242, 260

- friendly 165

- frowning 279

- gleaming 277

- glittering 218, 219

- goat-like 261, 330

- green 332, 459

- grey 155, 166

- grim 261

- hollow 221, 329, 332, 372, 408, 409, 416, 421

- inactive 330

- large/big 217, 218, 219, 221, 329, 330, 372, $374,395,396,408,409,416,421$

- left or right 66

- marvelous 168

- medium-sized 343, 374, 396, 399, 408, 416

- moist 247, 343, 410

- moving (constantly) 396

- open (wide) 89, 242

- pale 279

- piercing 330, 374

- protruding/bulging 329, 332, 374, 396, 416

- puffed-up 242

- pure 343, 410

- red $128,331,374,396,416$

- rigid/stiff 272, 273, 330, 332, 396

- rolling of 274

- saffron-coloured 331

- sharp(-sighted) 242, 256, 330

- shifty 168,416

- shining 279

- slit/squinty 374,416
- slow 330

- small 220, 330, 332, 416, 459, 460

- speckled/with spots 331,416

- staring 273

- still 89

- sparkling 216, 273

- spinning 89

- straining 242

- sunken 220, 221, 416

- terrible 211

- torch-like 314

- trembling 273, 330

- turquoise 332, 374, 416

- quivering/shivering 273, 332

- unshaken 168

- upsetting 262

- whites of 294

- yellow 331, 332

Eyebrow(s) 102, 164, 165, 168, 170, 171, 215, 273, 326, 336, 391

- arched 154, 342, 408

- beautiful 261, 342, 459

- black 374, 396

- charming 154, 155

- cheerful 165

- coming together above the nose 168 , 273, 459

- fine 171, 459

- hairy 333, 336, 374, 396, 417

- laid over iris 102

- locked together 273

- long 333

- medium-length 374, 396, 417

- mobility 198

- noble 168

- raised 273

- runs down side of nose 333

- straight 168

- stretches to temple 333, 374, 396, 417, 459

- thin 333, 396, 417

- triangular-curved 459

- twitching 89

- wide 459

- wide-apart 417

Eyelid 198

- broken 332

- joined 273

Ezekiel 306, 314 
Face $21,31,52,63,64,69,103,105,125,171$, 191, 198, 214, 215, 234, 240, 257, 266, 268, 336, 396, 397

- apsasû-bovine-like 101

- beautiful 372, 395, 408

- bison-like 101

- broad 12

- dark 101

- dog-like 101, 108

- easy 342, 408

- fox-like 101

- fleshy 335, 375, 397, 419, 460

- flushed 278

- full of holes 101

- full of pindû-moles 101

- full of ugudilû-moles 101

- full of umșatu-moles 101

- hideous 220

- honest 218

- in melothesia 126

- large/big 335

- lightning-like 314

- lion-like 101

- long 336, 375, 397, 419, 460

- mongoose-like 102

- noble 217

- pale 379

- Pazuzu-demon-like 101

- peeling 273

- pig-like 101

- pleasant 409, 421

- red 101, 216, 394

- repulsive 220

- round/oval 335, 343, 395, 399, 408, 410

- sallow 419

- scorpion-man-like 101

- scratched 174

- severe 217

- slender 419

- slim 460

- small 335, 397

- spasms of 273

- square 247

- stern 216

- streaked with yellow-green 101

- thin 335, 375, 397

- triangular 460

- ugly 336, 460

- yellow 375, 397
Face of a mongoose 102

Facial expressions 283

Facial features of a woman 120

Falcon 104

Fatalism 247

Fate 240

Fatimid Ismaili theologians 366

Favorinus 243, 244, 245, 250

Fee 336

Feeding Dumuzi's Sheep 16, 17, 18, 36

Feet $34,45,66,68,103,127,128,174,326$, $381,390,400$

- bad 214

- broad 341

- fleshy 274, 341, 399

- hard 341

- large/big 399

- little 341

- medium-sized 342

- small 341

- soft 341,399

- thick 242, 378, 399

Female omens 55

Fenni 236

Fertility 55

Fiction 149

Fictional plot 150

Fihrist 436

Finger(s) 22, 197, 198, 199, 217, 403, 458

- clear separation between 411

- gesticulate with 217

- lank 373, 377, 395, 408, 409

- long 341, 343, 398, 399, 408, 409, 420, 422

- short 341, 377, 398

- slender 409, 422

- thick 341, 377, 398

- thin 343, 408

Firmicus Maternus 133

Flesh 235

Flood hero 295

Foot of an ox 102

Foot of Taurus 133, 136

Forehead 31, 50, 51, 65, 68, 70, 165, 292, 326, $396,435,449$

- broad 418

- convex 459

- crowned 209

- flat 334, 375, 397

- frowning 334 
- great 459

- high 459

- huge 273

- lined 45, 459

- large/big 334, 411, 415

- medium-width 334, 375, 418

- narrow 128

- prominent 418

- protruding 386, 397

- protuberant 334

- rough 273

- small 334

- throbbing 45

- wide 397

- wrinkled 84, 85, 334

Forgery 363

Foundation of his nose 101

Fox 101

Frame 16

Framing element 19

Freedom of will 247

Front 171

Gait 47

Galen 238, 247, 250, 251

Gallic 248, 264

Gandhāra 58

Garga 42, 45, 46, 46, 47, 51, 55

Gārgīyajyotișa 42, 46, 48, 58

Garland of Prince Kuvalaya, The 42

Gaul(s) 233, 259, 261, 262

Ǧawāmi al-iskandarāniyyīn ("Summaries of the Alexandrinians") 451

Gaze 166, 168, 240, 250, 253, 254

Gecko 103

Gellius 210

Gemini 129

Gender 336

Gender separation 45

Genesis 6-9 296, 305

Genitals $68,70,71,127,312$

Genres 25

Geography 236, 253, 256, 259

German 241, 242

Germania 236

Germanicus 205, 218, 223

Gesamtkunstwerk 25

Gestures 199

Gilgamesh 106, 295, 305
Glance 170

Goat 222

Good physique 168

Good will 187

Good looking 171

Goose 48

Gorgias 184, 186

Göttertypen text 11, 19, 20, 22, 23

Great Collection (of Astral Science) 42

Greek 132, 368, 375

Grid of conventions 37

Grids of physiognomic correspondence 196

Groin 102, 103

Growth of plants 115

Gudea 12, 29

Gujarat 42, 57

Haemorrhoids 77

Hair 65, 70, 107, 136, 156, 166, 167, 168, $170,173,215,250,251,252,257,259$, $261,278,282,290,336,386,396,403$, 415,459

- abundant 242, 246, 417

- armpit 22

- beard 460

- beautiful 408

- black 173, 220, 259, 325, 395, 396, 408, 417, 458

- blond 154, 165, 171, 262, 379, 381, 409, 415

- body $48,49,51$

- bristling 216

- brown 171

- bull 135

- bushy 396

- chestnut $373,396,421$

- coarse 328, 373, 386, 395, 458

- curly $51,52,53,54,55,58,233,250,259,343$

- dark 410

- dishevelled 65

- head 48, 51, 52, 55

- lank 343

- long $166,167,169,170,171,218,219,372$, 395, 408, 409, 421

- loose 277

- medium-length 165

- pale 247

- prickly 220

- pubic 51

- pulled 174 
- raised 458

- red 128, 233, 325, 343, 396, 408, 410, 421

- scarce 411

- short 168

- soft $247,328,373,386,395,417,459$

- sparse 22

- standing 458

- straight 233, 235

- thick 170, 250, 417, 458

- thin 404

- twisted 44

- unkempt 279

- white 250

- woolly 229, 258

Hairs on the head 51, 52

Ḥamīd al-Dīn al-Kirmānī 366

Hamstrings 336

Hand(s) 21, 31, 34, 53, 68, 103, 126, 127, 156, $169,174,256,279,296,313,389,399$, 403, 407, 445

- in action 277

- large 373, 395, 408, 409

- left and right 217, 244, 251

- lined 50

- long 377, 420

- medium-sized 410

- micro-movements 198

- moving 419

- open 343, 407, 408

- plays with $338,378,398$

- reach the knee $377,398,420$

- raised 89

- restless 216

- shaking 274

- trembling 89

- well-built 407, 411

Handy-Tables 369

Hatred 188

Hattuša 57

Head 12, 20, 21, 43, 44, 45, 49, 51, 52, 53, 54, $58,63,65,66,70,71,73,75,77,82,93$, $105,108,122,125,126,128,132,163,164$, $171,174,215,217,221,235,256,258,274$, $324,325,339,389,395,400,446,459$

- bald 251

- beautiful 173

- chameleon-like 84

- large/big 82, 458

- ox-like 102
- medium-sized 342, 343, 372, 399, 403, 408, 409, 410, 421

- moving 89

- proportional 458

- right and left side 52

- round 247

- shaved 238, 240

- small 420, 458, 460

- spinning 89

- turning 89

Head of an ox 102

Head to feet 128

Heart 105

Hebrew 375

Hebrew Bible 305

Heels 400

Helen 176, 176

Heliodorus' 267

Hellenic 268

Hellenicity 259

Hellenistic context 132

Hellenistic influences 125

Hemerology 124

Heracles 253, 254, 282

Hermogenes of Tarsus 5, 157

Herodotus 234, 245, 256

Heroicus 164,165

Hesiod 191

Hierarchy of pathe 148

Hierarchy 250, 251

Hindu 55

Hippocrates 161, 217, 250, 332, 333, 334, 394 , 395, 451

Hippocratic 243, 266

Hippocratic medical treatises 192

Hippocratic theory of climatic or environmental influences 234

Hippolytus 376

Hip/waist 69, 77, 127, 163, 314, 395

- fleshy 341,372

- medium 372, 395, 406, 408, 409

- non-fleshy $343,408,409$

- protruding 342

Hireling 123

Historiography 267

Home of the Fish 16

Homer 171, 174, 176, 185, 191

Homerica 177

Homo signorum 125 
Homosexuality 248

Hot-blooded 166

Hugo Ripelin 338

Human marks 49

Humoral medicine 1

Ḥunayn ibn Isḥāq 331, 371

Hunnic 264

Huns 259, 263

Hygiene 373

Hymn 29

Hypogastric region 77

Hyppolytus 176

lamblichus 240

latromathematical Calendar Texts 135

Ibn Abī Ușaybi'a 376

Ibn al-Bițrīq 372, 377, 436, 441, 442

Ibn al-Nadim 436

Ibn Juljul 371, 435

Ibn Muqla 360

Icon parodies 305

Iconosphere 246

Ideal vizier 414

Ideal warrior 55

Identities 255

If-then 46

Iliad 172

'Ilm al-haqā'iq ("Science of the true meanings") 448

Imagines 14, 154, 155, 164, 201

Imbalances 251

Impiety 246

Impotence 245

Improvisation 186

Indexical iconicity 37

India 6, 42, 58

Indian medicine 42

Indian omen texts 41

Indian physiognomy 42

Indian system of human marks 41

Indian verse 46

Indus Valley 57, 58

Inference from signs 240,252

Inlustratio 152

Insane because of excessive drinking 281

Insanity 275

Institutio oratoria 151, 183

Interlinear commentaries 84

Interpretation of Dreams 164

Investitures 82
Irony 253

Irrationality in rhetoric 186

Irrefutable signs 186

Isaac Porphyrogenitus 161, 162, 164, 176

Ištu muhhi adi šēpe 128

Ius imaginum 207, 208

Jai (Prakrit) 54

Jain Uddyotana 43

Jassa (Prakrit) 54

Jaws 101

Jerking and moving blood-vessels or sinews 83

Jesters 240

Job of Edessa 442

Johannes Philoponos 451

John Malalas 161

John the Grammarian 436

Judah 309

Judgement 243

Julian 258

Julio-Claudian dynasty 217

Jyotihśsāstra 42

Kalendertext 129

Kalendertext scheme 134

Kalos 154

Kaloskagathos 172

Kamūnu-fungus 105, 111

Kataduggû 82, 84, 86, 91, 93, 94, 114

Kinship 176

Kirta 292, 297, 312

Kitāb al-Ghālib wa-al-maghlūb ("The Victor and the Vanquished") 376

Kitab al-Muwashsha 359

Kitāb al-Qānūn 367

Kittabru-moles 83

Knowledge 236, 243

Knowledge-creation 252

Knowledge-ordering 237

Kuvalayamālā 42, 53, 54

Lack of modesty 246

Lamassu 123

Lameness 245

Laodicea-on-the-Lycus 259

Large extremities 146

Large limbs 246

Late Babylonian astrology 123

Late Babylonian Uruk 84 
Late Harappan Period 57

Latin theorization of public speaking 188

Lavater 183

Legs $69,127,326,336,381,390$

- bow(-legged) 214

- bronze-like 314

- crooked 163

- fleshy 242

- medium-sized 410

- spindly 221

- straight 247

- thick 242, 341, 421

- thin 220, 222, 259

- weak 233

Leiden Polemo 244

Lemmata $16,18,19,25,36$

Leonine ideal 259

Leontius 258, 259

Lesion 76

Less intelligent 167

Laocoön 313

Letter of Pythagoras to Telauges 376

Lexical lists 16, 191

Libanius 265

Liber Legum Regionum 247

Libra 128

Ligurian 234

Lingua franca 56

Link between pathos and ekphrasis 150

Lion(s) 48, 101, 146

Lips 6, 65, 70, 102, 108, 198, 387, 435

- crab-like 102

- full of colour 155

- medium-thickness 418

- quiver 216

- red 400,418

- rough 273

- slim 242

- thick 335, 375, 397, 400, 418

- thin 247, 336, 397

- unclean 312

Literary portraits of madmen 280

Local identities 268

Logograms that are written in Emesal 121

London Physiognomy 357, 401

Loxus 241

Lucian 153, 252, 264

Lugalbanda 6, 33, 34, 35, 36, 37

Lumāšu 134
Macaon 176

Macroscopic 247

Madhya Pradesh 42, 43

Madmen 275

Maenad 156

Malalas 162, 164, 177, 176

Malformations in human and animal foetuses 67

Mania 274, 278

Manic type 278

Manifestation 11, 13

Manzalaoui 359, 437

Mapping observed entities into linguistically mediated descriptions 2

Marduk 130

Mark 76

Marriage(s) 55, 82

Mars 129

Marsyas 259

Marzubani 359

Masks of the ancestors 207

Mašmaššu 57

Massagetae 264

Mathematical arts 260

Maximinus Thrax 257

Maximus of Tyre 230, 248

Medes 57

Medical incantations 128

Medical writing 251

Medicine 238, 333, 334

Melancholy 274, 281

Melothesia 119, 123, 124, 132, 133

Menander Rhetor 254, 265

Menelaus 171

Mental disorder 277, 283

Mentally disabled child 105

Mesopotamia 6,191

Messages sent by the gods 3

Meta-iconic theorization of iconism 5

Micro-correspondences 200

Micro-zodiac 124, 129

Milinda's Questions 49

Mimetic 232, 243, 313

Mirror for princes 333, 337, 338, 435

Mise en abyme 37

Modalities of paradigmatic description 18, 37

Modes of persuasion 187

Modularity 7

Mole(s) 49, 76

Moon 129 
Morality 195

Mos maiorum 210

Mouse 103, 104

Mouth $30,31,65,70,89,90,93,104,108,114$, $172,312,313,314,326,333,336,381,387$, 396,397

- broad 242

- large 400, 418

- loose 242

- medium-sized 399, 408, 410

- open 152, 273

- small 165

- soft 155

- turns to the right 103

- wide 335, 375, 397, 418

Mouth-opening ritual 13

Muhammad Ibn al-'Alqami 359

Mucus 77

Muḥammad al-Dimashqī 447

MUL.APIN 123

Mungo 104

Music 26, 145

Mythology 162, 253

Nagasena 49

Nanše and the Birds 17, 18, 36, 37

Nasal turbinates 77

Nature 236

Navel 51, 64, 66, 70, 71, 77

Neck 17, 68, 70, 126, 165, 169, 171, 199, 218, $326,336,380,436$

- broad 342

- fine 460

- hairy $329,373,395,417,459$

- long 242, 339, 376, 397, 420, 460

- medium-length $372,395,408,460$

- movement of 115, 197

- short 339, 376, 397, 420, 460

- soft 460

- straight 343, 399, 408, 409, 421

- stiffly-held 217, 218, 219

- strong 247, 339

- swelling 260

- thick 261, 339, 410, 420, 460

- thickly-veined 397,460

- thin 220, 221, 242

- well-proportioned 400, 420

- woman-like 242

- upturned 273
Neo-Assyrian period 57

Nero 215, 217

Nestor 165

New Year's (day) festival 34, 296

Newborn child 77

Nicolaus the Sophist 5, 157

Nigdimdimmû 82, 84, 86, 92, 94, 114

Nimble 165

Nineveh 43

Ninurta 104

Nipples 64, 65, 69, 77

- of pregnant woman 76,77

Noble man 55

Non-verbal communication 197

Normative 251, 259, 267

Northwest Semitic 289

Nose $63,65,68,69,70,101,102,107,108,128$, $154,164,165,168,170,171,326,333,336$, $386,396,435,459$

- aquiline/eagle-like 101, 166, 170

- beautiful 343

- crinkled 102

- curved 102

- drooping 165

- even 102

- filled 333

- flat $168,333,375,396$

- in-between 101

- long 102, 333, 375, 396, 418

- movable 102

- noble 102

- open 334

- rises in the middle 418

- shape of 165

- snub 459

- straight $166,168,247,343,410$

- thick 375, 396

- thick in the middle 334

- thin 375, 396, 418

- thin-tipped 333

- turns to the right 103,109

- with divided foundation 101

Nostrils 75, 76, 77, 101, 103, 171, 198

- appropriate 459

- back of 101

- chubby 459

- close to mouth 418

- collapsed 103

- extended 418 
- flared 171, 375, 396

- long 396, 418

- pushed in 109

- right and left 101

- speaking 101

- sunken 109

- tender 400, 418

- wide (open) 396, 418, 459

Notional ekphrasis 4

Notional monoculture 259

Obituaries 257

Occult sciences $333,337,373$

Octagon of Justice 435,438

Odyssey 172

Ogmios 253

Oikoumene 236

Omen-driven sciences 3

Omens 1, 115, 121

On the Sublime 150, 152

Onomancy $373,374,375$

The Opinions of the People of the Virtuous City (Mabādi' ārā' ahl al-madīna al-fãdilā) 365

Opinions of the Philosophers 365

Orator 183

Oratory 241

Organon 192

Orion 130, 131

Overlap of physiognomic and behavioural omens 114

Ox 101

\section{Palaestra 199}

Pakistan 42, 58

Palamedes 176

Paleo-Hebrew 132

Palladios of Alexandria 451

Pallor 187

Palm(s) 50, 326, 336, 407

- delicate 340

- lank 409

- large 422

- long 341, 398, 420

- medium-sized 342

- reaches the knee 340,420

- short 341

- smooth $399,408,421,422$

- soft 340

- ugly 341
Panther 222

Parable of the Rich Fool 94

Paradoxical portrait 206, 207

The Parasite: That Being a Parasite is an Art 153

Parthian Empire 57

Passions and characters 188

Pathemata 154

Pathognomics 183

Pathognomik 209

Pathos 146, 147, 148, 150, 151, 158

Pathos-based interrelation 147

Pathos-centered relationship between physiognomy and ekphrasis 158

Patient 77

Paunch 31, 34, 213

Pausanias 259

Pazuzu-demon 101, 106, 115

Penelope 172

Penis $68,69,70,74,75,76,93$

Pergamon 255, 256

Periegesis 256

Persians 259, 263

Persuasion 184, 232, 248, 266

Peter Valvomeres 258

Phaedra 176

Phaedrus 207

Phalanges 199

Phantasia 150, 277, 283

Phantasiai 151

Phantasiai 152

Phasians 235

Phasis 234

Phenotypes 239

Philosopher-king 367

Philosophers 153

Philostratus 155, 156, 157, 164, 201, 250, 278,279

Philostratus the Elder 154, 156, 282

Philostratus the Younger 282

Phlegmatic 274

Phorbales 176

Phrazein 11

Phrenitis 274

Phusika pathemata 146

Physical deformities 191

Physiognomic commentary 121

Physiognomic omens 1

Physiognomic roots of Roman rhetoric 183

Physiognomic standard series 81,82 
Physiognomical analogies 241

Physiognomonica 45, 183, 194, 241, 257, 274, 276

Physiognomy 1-7, 41-59, 107, 113-116, 119-137, $143-158,161,162,164,171,176,196,197$, 199, 203-212, 222, 227-266, 271, 276, 285, $287,290,321-328,347-433,443-450$

Physique 235

- flabby 243

- fleshy 243

- good 168

- large 233, 235

- stocky 233

- strength of 168

- superb 290

- trim 166

- well-built 165

- well-nourished 235

Physique du role 172

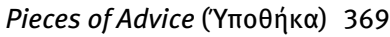

Pig 101

Pigmented naevus 76

Pindû-moles 101

Plato 184, 186, 415

Pliny the Elder 210, 236

Pliny the Younger 208, 211

Plutarch 206

Podalirius 176

Poetics 149, 150, 152

Polemon 46, 200, 225, 242, 243, 245, 250, 257, $259,332,333,334,394$

Pollux 176

Polybius Rhethor 162, 164

Polyxena 176

Portrait 208, 210, 214, 216, 217, 221

Posthomerica 177

Practical intelligence 187

Prakrit 41, 42, 47, 49, 51, 53, 54

Pregnancy 72, 76

Prejudices 233, 259

Preparation of drugs 77

Presencing 5, 14, 20, 26, 36

Pre-zodiological stage of melothesia 124

Priamus 171, 176

Prior Analytics 145, 147, 148, 187

Probabilistic knowledge 146

Problemata 274, 378

Progumnasmata 143, 157, 186, 231, 232, 254

Prolalía 254

Properties of stones, plants and animals 373
Propositional form 290

Propp 314

Prosopopoeia 232

Protasis 52

Proto-racist 251

Proverbial 230, 242

Province 240, 254, 257, 266, 268

Provincial ethnonym 254

Pseudo-Ammonius 365

Pseudo-Aristotelian letters to Alexander 415

Pseudo-Aristotle 183, 194, 220, 222, 242, 334

Pseudo-Galenic Ad Pisonem 436

Pseudo-Hippocratic Letters 367

Pseudo-Hippocratic Signs of Death (fí 'alāmāt al-mawt) 441

Pseudo-Longinus 150, 152, 157

Psychosomatic 256

Ptolemy 238, 239, 246, 247, 248, 260, 261, 369

Publius Rutilius Lupus 162, 164

Pupils 294

- lapis lazuli-like 293

- tend toward whiteness 331, 333

Purāna 42, 48

Pythagoras 161, 240, 250

Quintilian 1, 151, 152, 153, 157, 183, 184, 185, $188,192,194,197,200,201,217,248$

Qumran 131, 132, 133, 136, 137

Racial models 2

Racist 251

Rajasthan 42

Rapid movement of the tongue 273

Raven 48, 104

Razi 398

Recipes 3

Refutation of All Heresies 376

Regions 255

Relative pronouns 54

Relative-correlative construction 54

Repetitive frame 18

Representation 11, 13

Republic 415

Res gestae 257

Retrospective diagnosis 78

Return of Lugalbanda, The 33, 35

Retuning text, Old Babylonian 26

Rhetoric 186, 240, 241, 251, 256, 258, 267

Rhetorica ad Herennium 185, 197 
Rhetorical 'demonstration' 186

Rhetorical handbooks 290

Rhetorical theory 1, 144

Rhetoricians 153, 158, 252

Rhetorius-Teucer text 133

Rhodogoune 6,154, 155

Ribs 31, 69, 126

- delicate 339

- fine 460

- fleshy 339,460

- light 460

- strong 339

- thin 339

- tight 460

Romani language 51

Romanitas 259

Royal hymns 24, 25

SA.GIG (Sakikkû) 43, 72, 81, 85, 93, 107, 124, 128,131

Sagittarius 128

Sallust 206

Sanskrit 41, 42, 47, 49, 51, 56

Sardinians 234

Śārdūlakarṇāvadāna 49

șâtu-commentary 121, 122

Saturn 129

Schemata dianoia et lexeos 164

Scipio 212, 223

Scopas 156

Scorpion 104

Scorpion man 101

Scribal academies, Old Babylonian 16

Scythian(s) 237, 242, 245, 246, 259, 264

Scythians 242, 245, 246, 259

Sebasteion 256

Second Isaiah 305

Second Sophistic 1, 143, 144, 153, 158, 201, 259, 290

Secretum Secretorum 331, 397

Sêmeia 186

Sêmeion 187

Seneca 210, 218

Sensory vividness 258

Serpents 258

Service of royal court 55

Sexual deviance 246

Shield of Achilles 5

Shin 68, 122, 127

Short 168
Shoulder blade 77

Shoulders $69,126,326,336,380,388,389,398$

- bent/bending 372, 395, 408, 409, 421

- blade 77, 129

- blades meeting at backbone 343, 408

- broad 171, 217, 218, 400, 420

- full 460

- hairy 329, 373, 395, 417, 459

- hanging down 460

- large 340, 377, 398, 410, 420

- prominence 398

- protruding $340,377,398,420$

- rounded 164, 229

- square 399, 408

- stooped 229

- thin 340

- towering-tip 340

- upraised 420

- wide 340

- without flesh 343

Siddharși 53

Sigla 449

Signs 186, 232, 245

Šikinšu texts 11

Similes that involve mythological beings 115

Simonides 191

Sindh 42

Sinister-dexter 54

Sirr al-asrār 331, 364, 369

Size 168,171

Skandapurāṇa's Kāśīkhaṇḍa 48, 49

Skin 173

Skin diseases 191

Skin lesion 72

Sleeping 103

Smile 282

Snake(s) 104, 105, 235

Snout of a dog 102

Social status 191

Socrates 334, 395

Soles 50

Solomon's building activity 306

Somatic 243

Sophists $184,185,186$

Speech 336, 398

Speechless 280

Spittle 104

Standard Babylonian Calendar 125

Statue of Medea 281 
Steps 336,399

Stereotypes 233, 238, 239, 241, 252, 268

Stoic 160, 367

Stone-plant-wood-scheme $125,126,132$

Stories 254

Subaltern 268

Suetonius 205, 214, 215, 216, 217, 218, 220 , $222,223,224,225$

Suetonius' biographies 212

Šulgi and Ninlil's Barge 15, 17, 18, 24, 25, 28, 29

Šumma alamdimmû 43, 44, 63, 72

Šumma ālu ina mēle šakin $67,85,86,110$, 113,114

Šumma amēlu muhhašu umma ukâl 75

Šumma Ea liballițka 85, 113, 114

Šumma izbu 66, 130

Šumma kataduggû 44, 48

Šumma liptu 44, 49, 83

Šumma nigdimdimmû 44

Šumma šer'ān pūt imittišu ittenebbi 45

Šumma șillašu kīma rīmi 114, 115

Šumma Sîn ina tāmartišsu andullu arim 121

Šumma sinništu qaqqada rabât 44, 64, 82

Summaria Alexandrinorum 451

Swabia 337

Syncretic Hymn to Ninurta, The 20, 22, 36

Syriac 368,375

Syro-Palestine 6

Tacitus 206, 236

Talismans 373

Talk (lalía) 254

Taurus 122, 123, 130, 131, 132, 134

Taxila 57

Technical literature 3

Technical writing 238, 267

Teeth 20, 108, 216, 387, 435

- adjacent 418

- broad 335

- canine 335

- even 336

- gnashing 94, 260

- good 397

- ill-kept 213

- long 335, 397

- prominent 400,418

- protruding 418, 460

- scattered 335

- slightly-spread 418
- small 213

- straight 400,418

- strong 335

- thin 335

- turned around 102

- weak 335

- wide-apart 213, 460

Teichoscopy 171

Tekmêria 186

Tekmêrion 187

Temple ideology 296

Terminus technicus 134

Testicle 68,70, 242

Theophany 2, 4, 33, 34

Therapeutic texts 61,73

Thersites 171, 177

Theurgy 373

Thighs $68,69,127,261,389,399$

- fleshy 421

- not corpulent 409, 421

- not full 399,408

- thick 421

Thracian(s) 241, 242, 259

Tiamat 106,130

Tiibb al-Manșūrī 398, 412

Tiberius 205, 218, 220, 221, 221, 223

Tiger 48

Tigi Hymn(s) 4, 6, 11, 14, 15, 23, 25, 26, 27, 28, $29,33,36,37$

Timagenes of Alexandria 261

Tisias 185

Tongue 22, 41, 65, 66, 68, 156

- rapid movement of 273

Topos 231, 243, 248

Torah 309

Toughness 16

Towering 166

Transgender 245

Transmission 41

Triplicities 128

Triumph 236

Trojan War 176

Troy 171

Trypho Grammaticus 162, 163

Twins 129

Type-scene 34, 297

Tzetzes 162, 164, 176, 177

Tzetzes Grammaticus $161,162,164$, 176,177 
Uddyotana 53

Ugallu-hybrid creature 116

Ugarit 292, 297

Ugudilû-moles 101

Ugu-mu 128

Ujjain 42

Umāmu 144

Ummedu 73

Umșatu 61, 71, 101

Unusual births 67

Upamitibhavaprapancā kathā 53, 54

Upper arm 103

Ura/Urra 128

Urbane pronunciation 200

Urine 104

Ušumgallu-dragon-snake 116

Uta-Napishti 295

Vahāramihira 46

Valentinian 258

Valvomeres 259

Varāhamihira 42

Variety 247, 248, 250, 267

Varro 210

Venus 58

Vision-reports 317

Visual iconic media 1

Vitruvius 235, 236, 247

Vividness (enargeia) 11, 13, 148, 150, 151, 231, 233, 236, 247, 266, 267

Vocal pitches 236
Voice(s) 48, 165, 170, 197, 200, 233, 235, 336,398

Votive contexts 25

Votive object 4, 6, 14, 15, 23, 29, 35

Votive offering(s) 23,36

Warfare 333

Water-birds 48

Wealth 191

Will of the gods 50,77

Winter 12

Woman 103

World empire 230, 251

Yadi (Sanskrit) 54

Yasya (Sanskrit) 54

Year names 23, 24, 25

YHWH 309, 314

Zeno, the Great 365

Zenobia 257

Zimmernsches Secretum secretorum 337

Ziqqurrat 296

Zodiac man 125

Zodiacal animalia 136

Zodiacal melothesia 130, 133

Zodiacal physiognomy 119, 128, 129, 130

Zodiacal signs 122

Zoological method 193

Zoomorphic models 2

Zopyrus 334, 395 
